\title{
AÇÃO CORPORAL E AS REVERSÕES ENTRE CONSCIÊNCIA E MOVIMENTO: O REALISMO FENOMENOLÓGICO
}

\author{
Bodily action and the reversibility between consciousness and movement: \\ The phenomenological realism
}

Acción corporal y las reversiones entre consciencia y movimiento: El realismo fenomenológico.

\author{
Marcos Ricardo JanZen \\ Thiago Gomes DeCastro \\ WiLliam B. GOMES
}

\begin{abstract}
Resumo: O foco do artigo é a relação entre corporeidade e consciência para explicar como um ato que é pré-reflexivo e involuntário vem a se tornar reflexivo e voluntário, para se expressar posteriormente como pré-reflexivo e voluntário. Primeiro traça um paralelo entre psicólogos que recorreram à psicologia fenomenológica ou descritiva em experimentos sobre a consciência imediata dos sentidos. Segundo, ressalta o corpo como referencial ao movimento e à ação na constituição da autoconsciência, indicando divergências com teorias cognitivas e convergências com a fenomenologia existencial. Terceiro, toma-se o corpo situado interagindo com um mundo real para demonstrar que parte da apreensão fenomenal é concreta e situada. Por isto, se diz que a percepção é uma presentação e não necessariamente uma representação. Por fim, recorre-se a exemplos de estudos recentes em campos como a educação física, dança e ergonomia para sugerir aplicações fenomenológicas ainda pouco exploradas: como delineamento de layouts, planificação de ambientes e prevenção de acidentes.
\end{abstract}

Palavras-chave: Cinestesia; Consciência; Percepção de movimento; Reflexividade; Affordance.

Abstract: The focus of the paper is the relation between movement and consciousness to explain how a pre-reflexive and involuntary act becomes reflexive and voluntary, to further on express itself as pre-reflexive and voluntary. First, it traces a parallel among psychologists that used phenomenological or descriptive psychology on experiments about the immediate consciousness of the senses. Second, it emphasizes how descriptive phenomenology recognized the body as reference for movement and action in the constitution of self-consciousness, indicating convergences with existential phenomenology. Third, the situated body is taken in its interactions with the real world to show how phenomenal apprehension is concrete and situated. Thus, it understands perception as a presentation and not necessarily a representation. Finally, it resorts to examples of recent studies in fields such as physical education, dancing, and ergonomics, suggesting an applied field of phenomenology not much explored as designing layouts, planning environments and preventing accidents.

Keywords: Kinesthesia; Consciousness; Movement perception; Reflexivity; Affordance.

Resumen: El enfoque del artigo es la relación entre movimiento y consciencia para explicar cómo es que un acto que es pre- reflexivo y involuntario se vuelve reflexivo y voluntario para expresarse, posteriormente como pre-reflexivo y voluntario. Primero traza un paralelo entre psicólogos que recurrieron a la psicología fenomenológica o descriptiva en experimentos sobre la consciencia inmediata de los sentidos. Segundo, resalta el cuerpo como referencial al movimiento y la acción en la constitución del auto consciencia, indicando convergencias con la fenomenología existencial. Tercero, tomase el cuerpo situado interactuando con un mundo real para demostrar que parte de la aprehensión fenomenal es concreta y ubicada. Por eso, se dice que la percepción es una presentación y no necesariamente una representación. Por fin, se recurre a ejemplos de estudios recientes en campos como la educación física, danza y ergonomía para sugerir aplicaciones fenomenológicas todavía poco exploradas, como el delineamiento de layouts, planeación de ambientes y prevención de accidentes.

Palabras-clave: Cinestesia; Conciencia; Percepción de movimiento; Reflexividad; Affordance.

\section{Introdução}

Um dos aspectos da corporeidade e da ação é a consciência do movimento, sendo a consciência o movimento intencionado. O problema de interesse é como analisar a relação entre consciência e movimento, quando se considera: 1) a orientação da consciência pelo movimento, 2) a orientação do movimento pela consciência, e 3) a expressão do movimento independente da consciência. Esses aspectos ganham relevância quando se depara com situações desenvolvimentais, interventivas ou pedagógicas em sincronias entre movimento e consciência, por exemplo, aprender a dirigir um automóvel, a jogar basquetebol ou a dançar. A presente análise focaliza a sequência feno- 
menológica reversiva e transformadora (curvilinear) na qual: 1) um ato pré-reflexivo e involuntário; 2) torna-se reflexivo e voluntário; 3) para mais adiante se expressar como pré-reflexivo e voluntário. A relação entre movimento e ação se apresenta como condição primordial à consciência de nós mesmos, isto é, como eu sei que sou eu (DeCastro \& Gomes, 2011a). Para compor a análise: primeiro revisaremos as contribuições da psicologia fenomenológica ou descritiva para os estudos da consciência, resgatando uma corrente de pesquisa promissora e fértil que é a fenomenologia experimental; segundo mostraremos como a fenomenologia descritiva reconhece o corpo como referencial para o movimento e ação na constituição da noção de autoconsciência; terceiro, discutiremos as implicações conceituais do pareamento entre ação corporal e percepção para as teorias cognitivas no contraste entre representacionalismo (percepção mediada) e presentacionalismo (percepção direta); quarto, argumentaremos que a fenomenologia é de certo modo realista ao tratar de um corpo situado interagindo com um mundo concreto e independente; e por fim justificaremos com estudos recentes a função do movimento corporal e da cinestesia na constituição da consciência, ressaltado sua importância para políticas públicas sustentáveis, educativas, ergonômicas, ecológicas e solidárias.

\section{Psicologia Fenomenológica}

Entende-se por consciência a relação que se estabelece entre um ato de apreensão e um objeto apreendido, manifesto no imediato do que se vem a conhecer, e do saber o que se conhece. Esta seria uma justificativa ao uso do termo autoconsciência. $\mathrm{O}$ ato se constitui no direcionamento da consciência para o objeto ou conteúdo mental em modalidades perceptuais, atencionais, imaginativas ou evocadas. A relação ato-objeto conjuga a unicidade posicional, denominada de experiência de primeira pessoa, que é a emergência ou criação de sentido em um dado situacionamento espaço-temporal e tácito. Por espaço entende-se a contingência geral do lugar onde se vive em um dado momento. Por tempo entende-se a conjunção histórica do passado pessoal, das relações de presença e das prospectivas de futuro. Dessa forma se constitui o cerne do momento existencial, aquele no qual se vive e do qual se pode falar com exclusividade (tácito), pois só o agente (sujeito) pode dizer o que pensa e o que sente sobre algo (objeto). Essa breve descrição da consciência distingue sua especificidade ontológica, que é a ação conjunta e ao mesmo tempo distinta entre o que se pensa e o que se é pensado. Este foi também o projeto filosófico de Edmund Husserl (1859-1938).

Husserl (Petit, 2010) buscava uma filosofia primeira como refundação para as ciências naturais. Ele avançava por meio de exercícios lógicos transcendentais (as condições a priori) e tinha somente a si próprio como sujei- to experimental. Por meio de sua visão purista de ciência de rigor, Husserl afastava tentativas empíricas na fenomenologia. Entretanto, paralelamente à sua filosofia, também se desenvolveu uma psicologia empírica de pressupostos fenomenológicos. Esses estudos visavam uma descrição da experiência consciente, sem a imposição de categorias à priori.

DeCastro (2013) destacou uma sugestiva e intrigante aproximação fenomenológica na psicologia experimental ao longo do século XX, nas contribuições de Carl Stumpf (1848-1936), Georg Elias Müller (1850-1934), Oswald Külpe (1862-1915), David Katz (1884-1953), Max Wertheimer (1880-1943), Herbert S. Langfeld (1879-1958) e James J. Gibson (1904-1979). DeCastro ocupou-se das inovações experimentais que consideravam a condição holística e corpórea da consciência. Neste estudo, daremos continuidade a essa linha de argumento, destacando as influências da fenomenologia descritiva na formação destes psicólogos, as relações que se seguiram entre consciência e movimento, e por conseguinte entre consciência, corporeidade e ação.

$\mathrm{O}$ interesse por fenomenologia na psicologia experimental foi um desenvolvimento paralelo ao pensamento de Husserl, tendo em comum a rica influência de Stumpf. Anterior à fenomenologia de Husserl, Stumpf pesquisou empiricamente a experiência imediata do campo dos sentidos. Os experimentos foram realizados em acústica e musicologia, introduzindo variações nas condições sob as quais as propriedades do fenômeno apareciam. Os resultados e atinham-se às descrições dos atos ou funções da consciência imediata. Stumpf acreditava que a fenomenologia tinha de ser o estágio básico da pesquisa científica. Por meio da fenomenologia como psicologia descritiva Georg Elias Müller, na mesma universidade que Husserl, em Göttingen, realizou estudos empíricos com memória e teoria das cores. Por sua vez, Oswald Külpe, que havia sido aluno de Wundt, desenvolveu um programa de psicologia experimental baseada em fenomenologia descritiva e entendida como ciência de realidades (DeCastro, 2013; Spiegelberg, 1972).

A psicologia fenomenológica experimental teve continuidade nas pesquisas de David Katz, orientando de Müller que desenvolveu uma teoria sobre a percepção das cores, publicada em 1911, enfatizando a função da intencionalidade na constituição da espacialidade. Conforme o próprio Katz (1950), sua teoria sobre a percepção de cores foi a primeira com explicação integral e holista sobre a iluminação.

A visão integrada da percepção ganhou notoriedade com a publicação em 1912, por Max Wertheimer, do trabalho sobre percepção visual do movimento. Neste estudo sobre a aparência do movimento, Wertheimer demonstrou a hegemonia da experiência global e holística na apreensão perceptiva. A conotação fenomenológica do estudo estava no interesse pelas aparências ou fenomenalidades, isto é, como o movimento surgia na consciência. 
Os procedimentos experimentais incluiam a observação e a descrição, sem assumir uma teoria a priori. Como se sabe, Wertheimer foi orientando por Külpe e sua pesquisa foi publicada apenas um ano depois do trabalho de Katz mencionado acima.

A mesma orientação holística e fenomenal aparece na psicologia ecológica de James J. Gibson. Sua teoria da abordagem ecológica (Gibson, 1979) teve contribuições da descrição fenomenológica para a fundamentação empírica de um self corporal: o corpo como centro de referência perceptiva na ação. Seu doutorado foi orientado por Herbert S. Langfeld, orientando de Stumpf na Universidade de Berlim, em 1909 (Gibson, 2001). Uma das características do trabalho de Gibson era a descrição ingênua da experiência, isto é, valendo-se da suspensão de teorias prévias, mantendo a mesma orientação dos psicólogos alemães descritos anteriormente. Note-se que a noção de corpo como centro de referência perceptiva o aproxima da fenomenologia da percepção de MerleauPonty (1908-1961).

Modelos recentes, como a ciência cognitiva enativa (Thompson, 2007), reconheceram influência da psicologia fenomenológica e se assemelham aos modelos empíricos demonstrados acima. Esses modelos buscam compreender e explicar as atualizações constantes e operantes da percepção espacial e da propriocepção. Eles verificam a relação entre a intencionalidade e a concomitância da autoconsciência na ação. Trata-se de um sistema de atualização ativo ou operante, em organização continuada conforme as pistas ambientais.

\section{Consciência e Movimento Corporal: a Cinestesia}

Retornemos, agora, ao o foco central do artigo que é a ação corporal e as reversões entre consciência e movimento. A reversão entre consciência e movimento é facilmente compreendida na conjunção ato-objeto. Essa conjunção é descrita como a transição do que aparece como pré-reflexivo ou espontâneo (experiência consciente) para o que se atenta como reflexivo ou objeto da atenção (consciência da experiência). Sendo a consciência uma manifestação encarnada, como dizia Merleau-Ponty (1945/1999), o corpo e seus movimentos desempenham papel importante no dado pré-reflexivo, e daí se justifica o interesse em saber como os humanos percebem e exercem o movimento corporal (DeCastro \& Gomes, 2011a; Sheets-Johnstone, 2011). Husserl (Petit, 2010) recorreu ao termo cinestesia para designar a experiência viva da postura e da orientação motora dos órgãos da percepção e do movimento. Por meio da cinestesia pode-se ter acesso à experiência do indivíduo, uma experiência subjetivamente vivida que mantém o organismo constantemente informado da quase localidade do corpo (Petit, 1999). Na teoria da constituição fenomenológica (Husserl, 1907/2000), a cinestesia orienta a autoconsciência do sujeito na ação, sendo a vigília con- comitante à constituição do ato e da consciência de desempenhar este ato (DeCastro \& Gomes, 2011a). Contudo, a cinestesia ocorre somente por meio da autoconsciência do sujeito na ação. Sendo assim, tal condição pressupõe o realismo da presença carnal e concreta do agente em um mundo que o envolve permanentemente e sem o qual ele não existiria. Em contraste, não se pode afastar desta condição existencial às instâncias temporais (presente, passado e futuro) e cognoscentes (percepção, pensamento, imaginação, memória). Tal condição implicaria, sim, em certa idealidade. Posto desta forma tem-se um realismo e um idealismo mitigado e não radical (DeCastro \& Gomes, 2011a). Por isso a insistência em afirmar que a fenomenologia não é nem pura subjetividade e nem pura objetividade (Merleau-Ponty, 1945/1999). Em suma, a cinestesia informa o fluxo constitutivo temporal que é a consciência (Sheets-Johnstone, 2011).

Estudos sobre cinestesia (Brozzoli, Makin, Cardinali, Holmes, \& Farne, 2011; DeCastro \& Gomes, 2011b; Kalckert \& Ehrsson, 2012) vêm chamando atenção para duas posições filosóficas em disputa nas ciências cognitivas (Chemero, 2009). De um lado está a corrente dominante conhecida como representacionalista e computacional, e do outro a presentacionalista e eliminativista. Na primeira (Fodor, 1987) as representações são estados da mente que funcionam para codificar os estados do mundo. Na segunda, os estados mentais não são intermediados por representações, mas estão envoltos em um mundo, apreendendo-o diretamente. Para os eliminativistas as teorias psicológicas podem dispensar a noção semântica de representação, não sendo necessário recorrer a símbolos para caracterizar estados mentais em termos do que eles representam (Chemero, 2009).

Discussão semelhante ocorreu no início do século XX entre estruturalismo e o funcionalismo nos primórdios da psicologia científica. Wundt, (1832-1920) seguindo a tradição de Descartes (1596-1650) e continuada por Kant (1724-1804), entendeu que era necessário determinar a estrutura dos elementos na nossa vida mental, para só então tentar compreender sua função. Em contraste, William James (1942-1910), mais próximo ao pensamento de Carl Stumpf e sensível às contribuições da teoria da evolução, acreditava que atos mentais somente poderiam ser compreendidos a partir de suas funções (Chemero, 2009).

Para as ciências cognitivas representacionais, o pensamento é a relação entre o agente e a representação mental das coisas do mundo. Para o computacionalismo, uma das ciências cognitivas representacionais (Fodor, 1981, 1983, 1987), o pensamento é o processamento de símbolos internos que representam objetos externos. Dito de outra forma, pensar é manipular representações mentais. Para a teoria computacional clássica, costumeiramente chamada de GOFAI (good old-fashioned artificial intelligence), a cognição é uma manipulação de símbolos formais guiado por regras. 
Esclareça-se que as ciências cognitivas eliminativistas não são totalmente antirepresentacionalistas, mas priorizariam as representações voltadas para a ação, dirigidas pela ação do animal. Essa oposição ao representacionalismo ganhou projeção com a ciência cognitiva corporificada (embodied) (Varela, Thompson, \& Rosch, 1991; Turvey, 1992; Thompson, 2007), influenciada pela teoria ecológica de Gibson (1966, 1979). Essas representações voltadas para a ação são mais primitivas, visto que levam ao comportamento efetivo sem fazer uma separação entre as representações dos objetivos do sistema cognitivo e as representações do mundo. Ou seja, o sistema perceptual não precisa criar uma representação do mundo destituída de ação, que viria a ser usada pela ação para guiar o comportamento. Ao invés disso, as representações são direcionadas para as ações. Acreditase que o ambiente por si só é rico em oportunidades ambientais para o comportamento diretamente percebíveis e informações para guiar o comportamento (Gibson, 1979). Dessa forma, o processamento das representações é mais leve, visto que o ambiente processa e disponibiliza boa parte dos dados. Essas pistas ambientais ficaram conhecidas como affordances.

Na teoria eliminativista, a cognição não pode ser compreendida nem como espelho do mundo, nem externa às ações de um organismo e nem uma determinação da história de vida do indivíduo (Chemero, 2009). Esse entendimento aparece tanto no behaviorismo como na psicologia ecológica de Gibson. No behaviorismo, o comportamento é definido por classes de estímulos ambientais e de respostas funcionais presentes no organismo (Morris, 2009). Já na psicologia ecológica o comportamento é guiado por pistas ambientais disponíveis (affordances), diretamente percebidas, sem necessidade de representações mentais ou computacionais. Uma distinção entre a teoria ecológica e o behaviorismo é a noção de affordances, isto é, propriedades efetivamente presentes no ambiente, inicialmente livres de efeitos e independentes do organismo. ${ }^{1}$ Um desdobramento recente do eliminativismo é o movimento da Radical Embodied Cognitive Science (Chemero, 2009). Nesta vertente, a cognição corporificada é descrita por meio de ideias não-representacionais e esquemas não computacionais. A cognição e ação são entendidas como fenômenos corporificados e dinâmicos que não requerem representações mentais. As representações não são necessárias, pois a condição ecológica consiste no pareamento entre o animal humano ou não humano e o ambiente. Parte das informações está disponível no ambiente e o

\footnotetext{
Gibson denota que o termo Affordance é desdobramento do que Koffka chamou de caráter de demanda e Lewin chamou de Aufforderungscharakter - caráter invitativo, em que o ambiente convida à ação. A diferença é que nos gestaltistas há o caráter fenomenal, o convite é para um indivíduo. Em Gibson, as affordances não variam de acordo com as demandas do observador, elas estão sempre presentes para serem observadas. Dessa forma atrelam espécie e ambiente ao mesmo tempo, sem se prender a um dos pólos da relação (Gibson, 1979).
}

animal está especialmente orientado para receber essa informação das affordances. Esse pareamento ocorre nas situações cotidianas em um mutualismo operacional entre percepção e ação, ou seja, a ação ocorre concomitante à percepção (Chemero, 2009).

Os estudos sobre cinestesia tiveram algumas consequências para o grande campo da fenomenologia. Primeiro destacaram a importância do pareamento entre percepção e ação e, por conseguinte, a corporeidade da consciência. Segundo, o reconhecimento do pareamento entre percepção e ação levantou dúvidas sobre a noção de representatividade, como entendida em teorias cognitivas, por influências da tradição em processamento computacional. Esses interesses aumentaram consideravelmente nas últimas décadas, entre outros motivos, com o propósito de resolver impasses nas teorias cognitivas, como a falta de explicação para as relações diretas entre ambiente e ação. São exemplos, os estudos em neurofenomenologia (Varela, 1996) e a fenomenologia front-loaded (Gallagher, 2003). Trata-se de dois modelos para a realização de experimentos, onde ação e percepção são tradadas de maneira integrada e as experiências de primeira pessoa incluídas como dados para análise.

Ocorre que o pareamento entre ação e percepção levanta outro problema: a naturalização da fenomenologia. Críticas à naturalização procedem basicamente dos fenomenólogos transcendentais que tratam de uma intencionalidade voltada para ela mesma, em permanente conflito com o apriorismo da consciência. No entanto, reduzir o campo fenomenológico à consciência transcendental afastaria uma enorme e esclarecedora contribuição da fenomenologia descritiva e corpórea. A tradição fenomenológica ao rejeitar uma circunscrição unicamente material à consciência não nega sua naturalidade (Smith, 1999). Antes, faz uma distinção ontológica entre categorias formais (atos intencionais) e categorias materiais (objetos dados à consciência), as formais podendo controlar as materiais. A ampliação do conceito de naturalização e o entendimento de que categorias formais (intencionalidade) atuam sobre categorias materiais (objetos reais ou abstratos) estão na base da Neurofenomenologia, da Fenomenologia front-loaded, e em avanços recentes da fenomenologia experimental (ver Albertazzi, 2013).

\section{Realismo Fenomenológico}

O paramento percepção-ação como entendidos em tendências fenomenológicas recentes no estudo do movimento (Gallagher, 2006; Thompson, 2007; Gallagher \& Zahavi, 2008) fundamentou a explicação não representacionalista ou presentacionalista para consciência. Toma-se a relação direta entre percepção e ambiente, não se recorrendo a mediadores como mente ou representações. Temos então outro problema a considerar: o realismo fenomenológico. Neste realismo postula-se que: (1) ao 
menos algumas das percepções/pensamentos/teorias são corretas, e (2) que objetos das nossas percepções/pensamentos/teorias corretas existem em um mundo independente de nós. Sendo assim, é neste mundo independente que buscamos a confirmação das nossas percepções/ pensamentos/teorias.

Um exemplo desta tendência é o realismo de entidade. Neste realismo (Hacking, 1982, 1983) a existência de entidades teóricas é garantida pela nossa habilidade de manipulá-las durante um experimento. Isso ocorre quando entidades teóricas são usadas como ferramentas na investigação experimental de outras entidades. Hacking ilustra seu argumento, citando que o uso de elétrons em experimentos para investigar algo qualquer, digamos, bósons neutros, é também uma afirmação da crença em elétrons. Desde modo, quando os elétrons se tornam ferramentas do experimentador elas adquirem status de real, alinhando-se com os outros instrumentos utilizados no laboratório (como computadores, pipetas, e-prime, rubber hands, etc). As entidades teóricas ou conceituais se transformam em reais quando se tornam parte da prática do experimentador. quer essas entidades teóricas sejam conceituais ou reais.

O realismo de entidade aparece com clareza no Rubber Hand Illusion (DeCastro, 2013; Kalckert \& Ehrsson, 2012). Neste experimento, um membro fictício externo ao corpo é utilizado para verificar as noções de agência e pertença. Para isso, estimula-se uma mão de borracha concomitantemente à mão real. $\mathrm{O}$ participante tem em sua visão apenas a mão fictícia. Os resultados mostram que os participantes tendem a perceber o membro fictício como parte do próprio corpo, ou seja, no decorrer do experimento o participante passa a sentir a mão de borracha como sua própria mão. Por conseguinte, não é de se surpreender com enativistas e neurofonomenólogos como Thompson e Varela (1991) afirmarem que eventos neurais não são condições necessárias e suficientes para a experiência consciente. Os chamados "correlatos neurais da consciência” estão fadados a falhar, visto que a experiência consciente abrange o cérebro, o corpo e o mundo, por isto holística. Para Noë e Thompson (2004) a dificuldade de teóricos cognitivistas reconhecerem o presentacionalismo está em assumirem estados neurais como representações. Esses problemas são decorrentes das tradições teóricas que fundamentaram o movimento cognitivista e têm implicações ontológicas na concepção das atividades mentais, indo muito além das questões semânticas ou taxonômicas.

O realismo fenomenológico está fortemente associado ao conceito de affordances. Chemero, Klein, \& Cordeiro (2003) estudaram o efeito de pistas ambientais ou affordances na travessia de gaps (vãos, fendas, buracos, brechas ou descontinuidades) em um trajeto a pé. A hipótese era que relações estáveis entre medidas antropomórficas (altura dos olhos, comprimentos das pernas, flexibilidade), posturas (andando, correndo) e pro- priedades ambientais (tamanho da gap, profundidade do gap) indicariam se os humanos perceberiam ou não os gaps como obstáculos. Deste modo, o ajustamento das condições as características do sujeito e da tarefa mostraria quando as affordances apareciam e desapareciam para a travessia da gap.

Assim, temos a justificativa de dizer que as affordances possuem um caráter realista e demonstram que as experiências conscientes são aspectos genuínos dos sistemas corpo-ambiente. Deste modo, perceber ou guiar a atividade ao usar informações das affordances nada mais é que experienciar. Ademais, intencionalidade e consciência são inseparáveis, perceber algo de certa forma é inseparável de experienciar algo dessa certa forma.

\section{Movimento e Consciência}

Husserl (1907/2000) descreveu a cognição em termos de sua história imediata, de seus modos constitutivos corporais e situados. Nesta história imediata enfatiza a pertinência do movimento corporal inteligente atento às escolhas disponíveis no entorno situacional, compondo a unidade fluida de consciência. A cinestesia é uma vigília concomitante ao ato consciente e à consciência de desempenhar este ato (DeCastro \& Gomes, 2011a), pois focaliza a consciência no corpo e nos seus movimentos. Ademais, a cinestesia é um sentido que não pode ser desligado ou abafado voluntariamente. Podemos fechar nossos olhos e não mais ver, ou tapar as orelhas e não mais ouvir, fechar a boca e não mais sentir sabor, mas não temos como abafar ou cortar nossos corpos cinestésicos, a sensação do movimento (ou permanência) está sempre presente. Em casos extremos como a anestesia geral, simplesmente se desliga a consciência de si e do mundo.

O ser humano se movimenta livre e deliberadamente pelo mundo. A experiência humana diária inclui pensar no movimento (Sheets-Johnstone, 2010), como levantar da cama quando se acorda pela manhã, acionar a força necessária para levantar uma xícara de café matinal e calcular o tempo necessário para atravessar uma rua quando se sai para o trabalho ou para o exercício. Essa habilidade de pensar no movimento está baseada em conceitos humanos de espaço, tempo, energia e força, que por sua vez derivam da cinestesia (SheetsJohnstone, 2011).

O movimento acompanha o ser humano desde o período pré-natal, com o desenvolvimento gradativo da capacidade de percepção e de resposta corporal às demandas internas e externas do organismo (Gallagher, 2006; Petit, 1999). O movimento e a sensibilidade do sistema proprioceptivo contribuem para o desenvolvimento das estruturas neuronais, responsáveis não só pelo controle motor, mas pela consciência de si. O movimento está nas origens ontogenéticas que permitem um organismo ser autoconstitutivo. 
Desde o nascimento somos formas animadas, em movimento. Chegamos ao mundo nos movendo, não como um corpo e um mundo, não como uma pré-estrutura que virá a desenvolver, e nem mesmo como um eu movo (Merleau-Ponty, 1945/1999). O neonato não tenta se mover, não pensa em movimentos nem se coloca na tarefa de se movimentar. Ele se movimenta, não é uma propensão ao movimento, mas o movimento em si. A partir do movimento vivemos e fazemos sentido do mundo. Não ocorre um controle do corpo, mas sim uma descoberta do corpo a partir do próprio movimento. Uma criança não é uma mente tentando controlar um corpo nem um corpo fora de controle esperando por uma mente o alcançar. Crescemos cinética e cinestesicamente para dentro do nosso corpo, descobrimos a nós mesmos por meio do movimento, fomos aprendizes dos nossos próprios corpos, descobrimos braços que esticam, joelhos que dobram, boca que abre e fecha e que mastiga. Disso decorrem conceitos humanos tais como esmagamento, dureza e afiadez. O descobrimento das possibilidades corporais é resultado de ter se movido anteriormente. Logo, a cognição humana começa a partir do movimento (Sheets-Johnstone, 2011).

Conceitos espaço-temporais são fundamentalmente constituídos por meio da nossa experiência de automovimento, que incluem chutar, esticar, alcançar, sorrir, engolir, etc. Nesse sentido, a cinestesia é uma consciência de tempo é espaço. Por meio da experiência do movimento são criadas dinâmicas espaço-temporais. Ela nos provê dados imediatos desta dinâmica, elevando ao centro experiencial da consciência. A consciência cinestésica traz consigo qualidades que são experienciadas e incorporadas, nos dando a consciência de tempo e espaço. Tal assertiva é facilmente constatada em termos como fluxo, fluir, corrente de pensamento, claramente baseados em sentidos temporais e espaciais. O mais interessante é que estes termos usados para os mais diversos processos decorrem originariamente do nosso movimento espontâneo e não dos objetos. Ações de virar, andar, engatinhar, pular, bater, tremer são experiências que ensinam a diferença espacial entre o aqui e o ali, entre o anterior e o agora, entre o que vivo hoje e o que virá depois. Trata-se de experiências comuns a todos os seres humanos que compartilham o mesmo passado, o mesmo solo que não é feito de palavras, mas de um repertório de gestos e movimentos (Sheets-Johnstone, 2011).

O movimento envolve tanto a percepção da dinâmica exigida quanto à dimensão afetiva do controle voluntário (Sheets-Johnstone, 2010). A relação bidirecional entre consciência e ação do movimento vem sendo estudada para esclarecer tomada de decisão (Raab, Johnson, \& Heekeren, 2009), e a decodificação da informação espacial (Hölscher, Shipley, Belardinelli, Bateman, \& Newcombe, 2010). Estuda-se o comportamento espacial de agentes tanto biológicos quanto artificiais, e os processos de processamento que permitem o planejamento através de escalas temporais e espaciais. Essa informação espacial é crítica para coordenar a ação: agentes interagindo com objetos e se movendo entre eles; percebendo as relações espaciais, aprendendo sobre essas relações, agindo sobre elas ou armazenam informações para uso posterior, seja por meio deles próprios ou comunicando para outros. A pesquisa nesse campo tem envolvido psicologia e ciência da computação, que considera a natureza da informação como presente no mundo e tem uma consideração formal de como esses problemas podem ser solucionados. A pesquisa na cognição espacial humana também envolve a aplicação de representações e processos que podem ter evoluído para lidar com objetos e informações.

Estudos (Kunde, Skirde, \& Weigelt, 2011; Sebanz \& Shiffrar, 2009) que envolveram movimento e habilidades específicas mostraram que a proficiência de movimento que experts possuem dá a eles maiores habilidades para lidar com seu mundo específico, conseguindo reagir de forma mais adequada ao momento. Jogadores de basquete mais habilidosos conseguem fazer uma leitura mais eficaz se alguém irá jogar a bola em falso ou não.

Em contraste, cognitivistas como Bläsing, Tenenbaum, e Schack (2009), e Schack, $(2004,2010)$ explicam os movimentos como representações mentais. Tais representações mentais formam a base para uma redução funcional (voltada à tarefa em questão) de movimentos disponíveis. Para eles, as representações ajudam a formar os padrões de interação, armazenando resultados perceptuais-cognitivos do processo de aprendizado como itens (representações de movimentos) na Memória de Longo Prazo. Como se pode constatar, o modelo cognitivo explana o processamento mental envolvido em atividades de movimento, mas, mesmo reconhecendo as implicações proprioceptivas e cinestésicas, não esclarece a reversibilidade entre consciência e ação.

\section{Movimento: Origens Primordiais da Consciência}

Conforme apontado por Nagel (1993), as ciências cognitivas têm dificuldade de oferecer uma explicação plausível de como a consciência surge na matéria. Searle (1992) argumenta que a principal tarefa de uma teoria da mente é explicar a consciência, e Sheets-Johnstone (2011) vai adiante afirmando que essa explicação deverá vir da história evolutiva da forma animada. Para a autora a consciência surge em formas orgânicas, obrigatoriamente animadas, acompanhando a complexidade evolutiva dos animais. As capacidades cognitivas simples aparecem juntamente com os movimentos simples: desde bactérias, que se movimentam em direção à comida até os seres humanos com controle volitivo e deliberacional do movimento.

O movimento é um fenômeno percebido pela bactéria, e é a fonte e o próprio conhecimento em si. A bactéria necessita ter "conhecimento" acerca do seu ambiente e dos seus atos (por meio de uma cinestesia) e conseguir 
ir em direção à comida para se alimentar. Tal "conhecimento" é o início da consciência nos animais. A consciência não está presente na matéria, como diriam materialistas eliminativistas (como Churchland, 1988), mas sim nos seres animados que podem se movimentar. Os seres animados são sensitivos ao seu próprio movimento e aos movimentos que ocorrem no mundo. É o que nos mostra a Joaninha Vermelha (Coccinella magnifica - dos insetos coleópteros) que consegue continuar andando em uma folha no meio do vento, sabendo que tem que se firmar na folha para conseguir continuar sua trajetória em direção ao alimento. O movimento é a língua materna de todas as formas animadas, bem como gesto é a origem da fala humana e suas linguagens (Merleau-Ponty, 1945/1999). Temos no movimento tanto um fato primordial como uma possibilidade perceptual primordial da vida animada e falante (Sheets-Johnstone, 2011).

\section{Movimento: Implicações e Estratégias de Respos- tas em Organismos Animados}

Considerações ontológicas implicam em considerações éticas, entendo-se ética como práxis ou aplicação. Assim, uma das implicações do mutualismo entre ação e percepção são estudos em ergonomia. Vilar, Rebelo, Noriega, Teles \& Mayhorn (2013) demonstraram a influência das affordances do ambiente (como claridade e largura do corredor) na escolha de rotas dentro de edifícios. Os resultados podem ser usados por profissionais para planejar rotas tanto em situações cotidianas (a rota da atração principal de um centro de convenções, fazer pessoas evitar rotas de serviço em hotéis), quanto em situações de emergência (deixar um ambiente em perigo em menos de três minutos).

Outra implicação do mutualismo ação-percepção é o desenvolvimento de softwares que propiciem o uso direto das funções desejadas. Um estudo de Filgueiras, Rebelo, \& Moreira da Silva (2011) analisou o uso diário de computadores por funcionários e notou a grande utilização do mouse. No entanto, para a produção de textos o uso constante do mouse é contraproducente, visto que a tarefa requer basicamente o uso do teclado. Seria útil o desenvolvimento de softwares com menor uso do mouse, por meio de affordances que indicariam ao usuário menor uso do mouse. Essa ideia também se aplica em outras interfaces, como smartphones, nos quais as funcionalidades do aparelho precisam ser de direto acesso aos usuários. O mesmo ocorre com muitos aplicativos e desenhos de site, cada qual trazendo a própria hermenêutica, o que requer muita tentativa e erro para alcançar proficiência. Outras aplicabilidades desses estudos expandem o potencial e segurança humanos, como ferramentas adequadas para determinadas tarefas, máquinas para recuperação fisioterapêutica, personal trainers adequando rotinas para cada cliente e dispositivos de segurança.

\section{Considerações Finais}

A presente análise teve como objetivo demonstrar como um ato pré-reflexivo e involuntário torna-se reflexivo e voluntário para vir a se expressar como pré-reflexivo e voluntário.

Primeiro ressaltou a contribuição da psicologia fenomenológica em experimentos que focalizavam a percepção com interesse em manifestações pré-reflexivas ou espontâneas, isto é, a experiência consciente. Neste sentido, contrastou dois diferentes modos de explicar o acesso cognitivo: o representacionalismo das teorias de processamento da informação (Fodor, 1987) e o presentacionalismo das teorias enativas (Varela et al., 1999). Argumentou-se que o conceito de representação foi um artifício teórico para explicar como os estados da mente lidam com os estados do mundo. No entanto, estudos da relação entre movimento e ação na constituição da consciência apontam para evidências de que nossa ação imediata no mundo é, sobretudo, direta, sendo a intermediação as condições realistas como apresentadas (Chemero et al., 2003; Filgueiras et al., 2011; Vilar et al., 2013).

Segundo, reconheceu que os movimentos antecedem e determinam a experiência consciente e só posteriormente vem a ser reconhecidos e controlados pela consciência da experiência. Em outras palavras, a experiência consciente constitui-se na ação corporal que é o momento vivido de se movimentar. A seguir, transforma-se progressivamente em consciência da experiência no controle voluntário do fluxo encadeado dos movimentos. A passagem da experiência consciente para consciência da experiência na ação corporal é exemplificada no aprendizado de tarefas manuais e esportivas.

Terceiro, conclui que a proficiência de movimentos ocorre em situações nas quais a pré-reflexividade e o controle voluntário são os movimentos determinados pelas affordances, conjuntamente às ações de grande destreza. Neste caso, a proficiência da consciência da experiência reverte para a agilidade espontânea e o estado de prontidão pré-reflexivo habilitando o organismo para responder a ações inesperadas. São exemplos, a proficiência na direção de um automotor, a resposta a lances difíceis no esporte, a defesa corporal frente a situações inesperadas, ou a criatividade estética nos movimentos de uma dança.

A presente análise descortina outro lado do movimento fenomenológico, distante das conjecturas transcendentais e engajado na relação real e cotidiana dos atos da vida, das vicissitudes mundanas, o que não deixa de ser uma situação justificadamente existencial. A nossa ação corporal frente ao mundo dependente da nossa presteza e sensibilidade frente às affordances, ou da clareza das affordances em nos sinalizarem pistas inteligentes e seguras ao nosso bem estar, segurança, e qualidade de vida. Uma fenomenologia prática sensível a situações concretas e reais no sentido de promover pedagogias mais efi- 
cientes, planejamento ambiental ergonômico, e políticas de prevenção de acidentes e segurança pública. Temos então uma fenomenologia prática capaz de revitalizar as ciências cognitivas com evidências empíricas de uma consciência naturalizada, mas não materializada, e de revigorar a existência com políticas públicas sustentáveis, ergonômicas, ecológicas e solidárias.

\section{Referências Bibliográficas}

Albertazzi, L. (Org.). (2013). Handbook of Experimental Phenomenology: Visual Perception of Shape, Space and Appearance. Chichester, UK: John Wiley \& Sons.

Bläsing, B., Tenenbaum, G., \& Schack, T. (2009). The cognitive structure of movements in classical dance. Psychology of Sport and Exercise, 10(3), 350-360.

Brozzoli, C., Makin, T., Cardinali, L., Holmes, N., \& Farnè, A. (2011). Peripersonal space: A multisensory interface for body-objects interactions. Em: M. M. Murray \& M. T. Wallace (Eds.), The Neural Bases of Multisensory Processes (pp. 449-466). Boca Raton: CRC Press.

Chemero, A. (2009). Radical Embodied Cognitive Science. Cambridge, MA.; London: MIT Press.

Chemero, A., Klein, C., \& Cordeiro, W. (2003). Events as Changes in the Layout of Affordances. Ecological Psychology, 15(1), 19-28.

Churchland, P. M. (1988). Matter and Consciousness: A Contemporary Introduction to the Philosophy of Mind. Cambridge, MA.: MIT Press.

DeCastro, T. G. de. (2013). Percepção e Autoconsciência: Modelos Experimentais na Naturalização da Fenomenologia. (Tese de doutorado). Universidade Federal do Rio Grande do Sul, Porto Alegre.

DeCastro, T. G. de, \& Gomes, W. B. (2011a). "Como sei que eu sou eu?" Cinestesia e Espacialidade nas Conferências Husserlianas de 1907 e em Pesquisas Neurocognitivas. Revista da Abordagem Gestáltica, XVII(2), 123-130.

DeCastro, T. G. de, \& Gomes, W. B. (2011b). Autoconsciência e ambiguidade perceptual cinestésica: experimento fenomenológico. Psicologia em Estudo, 16(2), 279-287.

Filgueiras, E., Rebelo, F., \& Moreira da Silva, F. (2011). Human-Computer Interaction in Office Work: Evaluation of Interaction Patterns Using Office Equipment and Software during Data Entry and Navigation. Em M. M. Robertson (Ed.), Ergonomics and Health Aspects of Work with Computers (pp. 40-48). Berlin, Heidelberg: Springer.

Fodor, J. A. (1981). RePresentations. Cambridge, MA: MIT Press.

Fodor, J. A. (1983). The Modularity of Mind: An Essay on Faculty Psycholgy. Cambridge, MA: MIT Press.

Fodor, J. A. (1987). Psychosemantics: the problem of meaning in the philosophy of mind. Cambridge, MA; London: MIT Press.
Gallagher, S. (2003). Phenomenology and experimental design toward a phenomenologically enlightened experimental science. Journal of Consciousness Studies, 10, 9(10), 85-99.

Gallagher, S. (2006). How the Body Shapes the Mind. New York: Oxford University Press.

Gallagher, S., \& Zahavi, D. (2008). The Phenomenological Mind $\left(1^{\mathrm{a}}\right.$ ed.). London: Routledge.

Gibson, E. J. (2001). Perceiving the Affordances: A Portrait of Two Psychologists ( $1^{\mathrm{a}}$ ed.). Mahwah, NJ: Lawrence Erlbaum Associates.

Gibson, J. J. (1966). The senses considered as perceptual systems. Boston: Houghton Mifflin.

Gibson, J. J. (1979). The Ecological Approach To Visual Perception. Boston: Houghton Mifflin.

Hacking, I. (1982). Experimentation and Scientific Realism. Philosophical Topics, 13, 71-87.

Hacking, I. (1983). Representing and Intervening: Introductory Topics in the Philosophy of Natural Science. Cambridge: Cambridge University Press.

Hölscher, C., Shipley, T. F., Belardinelli, M. O., Bateman, J. A., \& Newcombe, N. S. (2010). Spatial Cognition VII: International Conference, Spatial Cognition ( $1^{\mathrm{a}}$ ed.). Berlin: Springer-Verlag.

Husserl, E. (2000). A idéia da fenomenologia. Lisboa: Edições 70. (Original em língua alemã publicado em 1907).

Kalckert, A., \& Ehrsson, H. H. (2012). Moving a Rubber Hand that Feels Like Your Own: A Dissociation of Ownership and Agency. Frontiers in Human Neuroscience, 6.

Katz, D. (1950). Gestalt psychology: its nature and significance. New York: The Ronald Press Company.

Kunde, W., Skirde, S., \& Weigelt, M. (2011). Trust my face: Cognitive factors of head fakes in sports. Journal of Experimental Psychology: Applied, 17(2), 110.

Merleau-Ponty, M. (1999). Fenomenologia da Percepção (2a ed.). São Paulo: Martins Fontes. (Original em língua francesa publicado em 1945).

Morris, E. K. (2009). Behavior Analysis and Ecological Psychology: Past, Present, and Future. A Review of Harry Heft's Ecological Psychology in Context. Journal of the Experimental Analysis of Behavior, 92(2), 275-304.

Nagel, T. (1993, March 4). The Mind Wins! [Review of the book: The Rediscovery of the Mind, by Searle, J.]. The New York Review of Books.

Noë, A., \& Thompson, E. (2004). Are there neural correlates of consciousness? Journal of Consciousness studies, 11(1), 3-28.

Petit, J.-L. (1999). Constitution by movement: Husserl in light of recent neurobiological findings. Em: J. Petitot, F. J. Varela, B. Pacoud, \& J.-M. Roy (Eds.), Naturalizing Phenomenology (pp. 220-244). Stanford: Stanford University Press. 
Petit, J.-L. (2010). A Husserlian, Neurophenomenologic Approach to Embodiment. Em: Handbook of Phenomenology and Cognitive Science (pp. 201-216). New York: Springer.

Raab, M., Johnson, J. G., \& Heekeren, H. R. (2009). Progress in brain research. Mind and Motion: The bidirectional link between thought and action (Vol. 174). Amsterdam; Boston: Elsevier.

Schack, T. (2004). The Cognitive Architecture of Complex Movement. International Journal of Sport and Exercise Psychology, 2(4), 403-438.

Schack, Thomas. (2010). Building Blocks and Architecture of Dance. Em The Neurocognition of Dance: Mind, Movement and Motor Skills (pp. 11-40). London: Psychology Press.

Searle, J. R. (1992). The Rediscovery of the Mind. Cambridge, MA. MIT Press.

Sebanz, N., \& Shiffrar, M. (2009). Detecting deception in a bluffing body: The role of expertise. Psychonomic bulletin $\&$ review, 16(1), 170-175.

Sheets-Johnstone, M. (2010). Body and Movement: Basic Dynamic Principles. Em D. Schmicking \& S. Gallagher (Eds.), Handbook of Phenomenology and Cognitive Science (pp. 217-234). Dordrecht: Springer Netherlands.

Sheets-Johnstone, M. (2011). The primacy of movement. Philadelphia: John Benjamins Publishing Company.

Smith, D. W. (1999). Intentionality Naturalized? In J. Petitot, F. J. Varela, B. Pacoud, \& J.-M. Roy (Eds.), Naturalizing Phenomenology (pp. 83-110). Stanford University Press.

Spiegelberg, H. (1972). Phenomenology in Psychology and Psychiatry: A Historical Introduction. Evanston: Northwestern University Press.

Thompson, E. (2007). Mind in Life: Biology, Phenomenology, and the Sciences of Mind. Cambridge, MA: Harvard University Press.
Turvey, M. T. (1992). Affordances and Prospective Control: An Outline of the Ontology. Ecological Psychology, 4(3), 173-187.

Varela, F. J., Thompson, E., \& Rosch, E. (1991). The Embodied Mind: Cognitive Science and Human Experience. Cambridge, MA: MIT Press.

Varela, F. J. (1996). Neurophenomenology: A methodological remedy for the hard problem. Journal of consciousness studies, 3(4), 330-349.

Vilar, E., Rebelo, F., Noriega, P., Teles, J., \& Mayhorn, C. (2013). The influence of environmental features on route selection in an emergency situation. Applied Ergonomics, 44(4), 618-627.

Marcos Ricardo Janzen - Mestre e Doutorando em Psicologia no Programa de Pós-Graduação em Psicologia da Universidade Federal do Rio Grande do Sul (UFRGS) e Pesquisador do Laboratório de Fenomenologia Experimental e Cognição - LaFEC. Endereço Institucional: IP/ UFRGS. Rua Ramiro Barcelos, 2600 - Sala 123. CEP 90035.003. Porto Alegre/RS. Email: marcosjanzen@gmail.com

Thiago Gomes de Castro - Mestre e Doutor em Psicologia pela Universidade Federal do Rio Grande do Sul (UFRGS), Pesquisador do Laboratório de Fenomenologia Experimental e Cognição - LaFEC e Docente do Curso de Psicologia da Pontifícia Universidade Católica do Paraná (PUC-PR). E-mail: thiago.cast@gmail.com

William Barbosa Gomes - PhD em Psicologia pela Southern Illinois University; fundador e professor do Programa de Pós-Graduação em Psicologia da Universidade Federal do Rio Grande do Sul (UFRGS). Coordenador do Laboratório de Fenomenologia Experimental e Cognição - LaFEC. Endereço Institucional: IP/UFRGS. Rua Ramiro Barcelos, 2600 - Sala 123. CEP 90035.003. Porto Alegre/RS. E-mail: gomesw@ufrgs.br
Recebido em 31.10 .12 Primeira Decisão Editorial em 26.03.13 Aceito em 06.05.13 